\title{
Frontières
}

\section{Le suicide et les aînés au Québec}

Un défi pour l'avenir

\section{Brian L. Mishara}

Volume 12, numéro 1, automne 1999

Suicides, générations et culture

URI : https://id.erudit.org/iderudit/1074505ar

DOI : https://doi.org/10.7202/1074505ar

Aller au sommaire du numéro

Éditeur(s)

Université du Québec à Montréal

ISSN

1180-3479 (imprimé)

1916-0976 (numérique)

Découvrir la revue

Citer cet article

Mishara, B. L. (1999). Le suicide et les aînés au Québec : un défi pour l'avenir. Frontières, 12(1), 33-38. https://doi.org/10.7202/1074505ar
Résumé de l'article

Les aînés québécois, malgré de nombreux facteurs de risque et leur faible utilisation des professionnels en santé mentale et des centres de prévention du suicide, se suicident moins que d'autres groupes d'âge, sont les plus satisfaits de leurs relations familiales et de leur réseau et connaissent le moins de détresse psychologique. Cependant, les facteurs importants de risque du suicide (par exemple, les pertes) sont souvent présents. La génération actuelle des aînés diffère de la prochaine génération. Il faut prévenir une augmentation du suicide des aînés dans l'avenir, trouver des façons d'identifier les aînés à risque de la présente génération et offrir l'aide appropriée.
Ce document est protégé par la loi sur le droit d'auteur. L'utilisation des services d'Érudit (y compris la reproduction) est assujettie à sa politique d'utilisation que vous pouvez consulter en ligne.

https://apropos.erudit.org/fr/usagers/politique-dutilisation/ 


\section{Résumé}

Les aînés québécois, malgré de nombreux facteurs de risque et leur faible utilisation des professionnels en santé mentale et des centres de prévention du suicide, se suicident moins que d'autres groupes d'âge, sont les plus satisfaits de leurs relations familiales et de leur réseau et connaissent le moins de détresse psychologique. Cependant, les facteurs importants de risque du suicide (par exemple, les pertes) sont souvent présents. La génération actuelle des aînés diffère de la prochaine génération. Il faut prévenir une augmentation du suicide des aînés dans l'avenir, trouver des façons d'identifier les aînés à risque de la présente génération et offrir l'aide appropriée.

Mots clés: suicide - aînés - différence générationnelle - prévention

\section{Abstract}

Québec's elderly, despite numerous risk factors and their low use of professional mental health services or suicide prevention centers, commit suicide less frequently than all age groups, are the most satisfies with their family relationships and social network, and suffer the least psychological distress. However, important suicide risk factors, e.g. losses, are often present. The current generation of the elderly differs from the coming generation. A future increase in suicide among the elderly must be prevented; ways of identifying the elderly at risk in the current generation have to be found; and the proper assistance needs to be offered.

Key words: suicide - elderly - generational differences - prevention

\section{Le suicide et les aînés au Québec Un défi pour l'avenir}

\begin{abstract}
Brian L. Mishara,
professeur au Département de psychologie, UQAM, directeur du Centre de recherche et $d^{\prime}$ 'intervention sur le suicide et l'euthanasie (CRISE).
\end{abstract}

Dans presque tous les pays du monde, les taux de suicide des hommes âgés sont les plus élevés de tous les groupes d'âge et, parmi les femmes, ce sont le plus souvent celles de 75 ans et plus qui s'enlèvent la vie. Cependant, tel n'est pas le cas au Québec. Ici, ce sont les personnes d'âge mûr qui se suicident le plus. Comment peut-on expliquer le fait qu'au Québec le suicide n'augmente pas avec l'âge? Est-ce parce que la vie est meilleure pour les aînés au Québec qu'ailleurs? Quelles sont les implications de ce constat pour la prévention du suicide des aînés et notre compréhension de ce phénomène au Québec?

\section{LE SUICIDE DES AÎNÉS}

Le tableau 1 indique le taux de mortalité par suicide au Québec en 1997 par groupes d'âge. Il est évident que ce sont les hommes âgés entre 20 et 59 ans qui ont les taux les plus élevés, plus particulièrement les hommes de 40 à 50 ans. Pour les femmes, les taux les plus élevés se situent entre 35 et 64 ans. Cependant, si on regarde bien le graphique, on constate un très haut taux chez les hommes de 85 à 89 ans. On ne doit pas se fier à ce taux élevé; il s'agit en fait de 8 décès dans l'année (voir tableau 2). Avec un si petit nombre de décès par suicide, dans la faible population des aînés, une différence de seulement 2 décès peut faire augmenter ou diminuer les taux de $25 \%$.

Tous les chercheurs, y compris l'auteur de cet article, s'entendent sur le fait que les taux de suicide des aînés sont sousestimés par rapport aux taux réels pour ce groupe d'âge. On sait que les coroners ont moins tendance à enquêter sur le décès d'un aîné déjà très malade, mort dans son lit et sans signes suicidaires, que sur le décès d'une personne plus jeune. On n'a cependant aucune raison de croire que cette tendance à la sous-estimation est plus élevée au Québec qu'ailleurs. En effet, une des explications pour les hauts taux de suicide au Québec en général tient à l'hypothèse que le bureau du coroner du Québec fait mieux son travail qu'ailleurs. La sous-estimation des taux de suicide des aînés est probablement universelle et donc n'explique aucunement les différences entre les taux relativement plus faibles des aînés au Québec et la croissance du suicide avec l'âge dans la plupart des pays. 


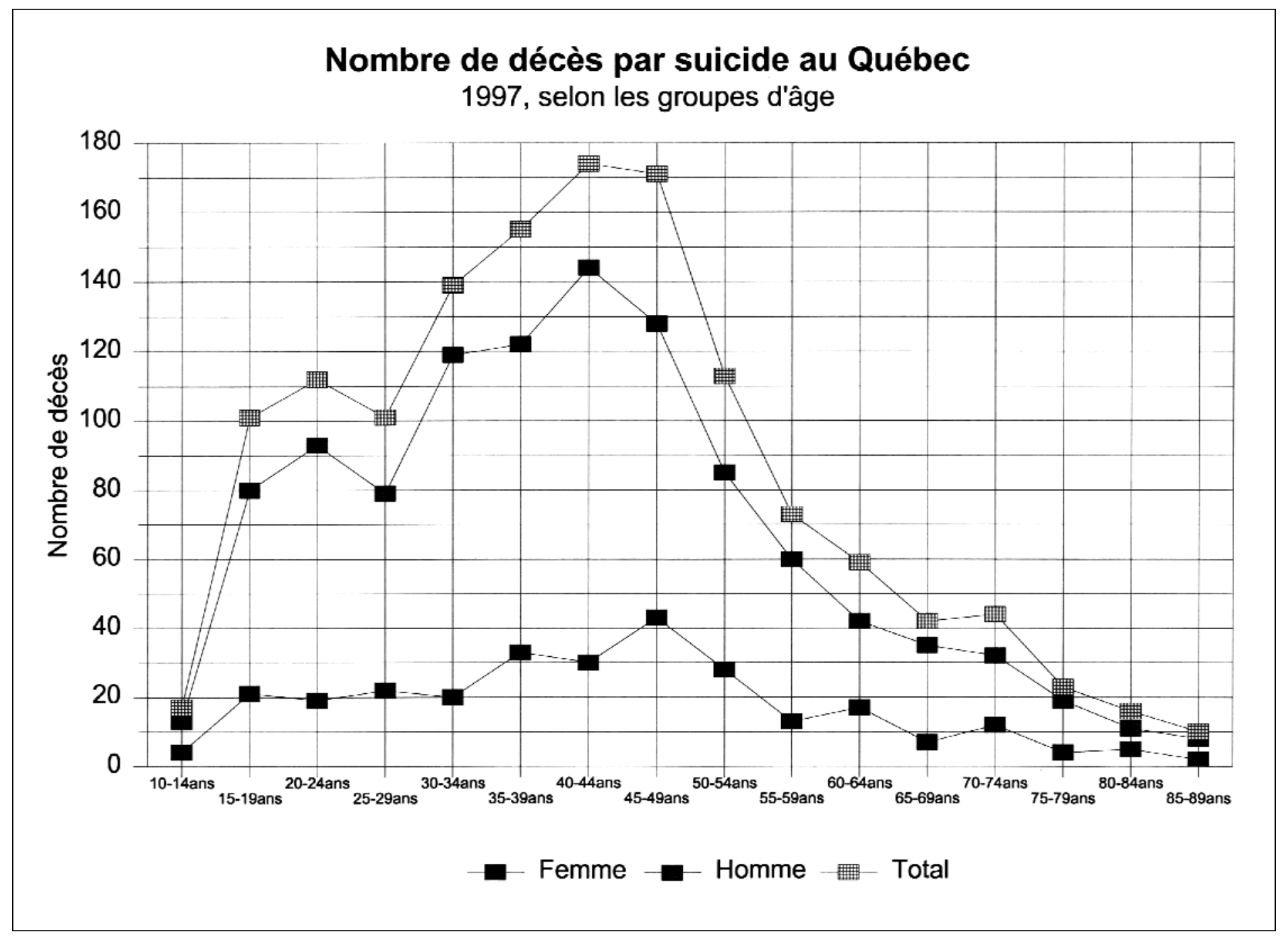

SOURCE : Danielle St-Laurent, MSSS, Direction de l'analyse et de la surveillance, février 1999

EXPLICATIONS DE LA MOYENNE MOINS ÉLEVÉE DES TAUX DE SUICIDE DES AîNÉS AU QUÉBEC

Les recherches épidémiologiques récentes indiquent que certaines générations ont tendance à connaître de plus hauts taux de suicide que d'autres ${ }^{1}$. Il y a plus de cinquante ans, quand la génération actuelle des aînés au Québec était jeune, les jeunes du Québec avaient un très faible taux de suicide. Une façon de comprendre le taux de suicide des aînés d'aujourd'hui est de le considérer comme un phénomène générationnel: la génération actuelle des aînés a toujours connu un faible taux de suicide par rapport aux générations subséquentes. Si on accepte cette logique, à moins qu'on ne développe bientôt des programmes de prévention primaire efficaces, on peut d'ores et déjà prévoir une augmentation importante des décès par suicide dans les prochains dix à vingt ans, lorsque la génération actuelle des baby-boomers, qui a toujours eu un taux de suicide rela- tivement élevé, formera la tranche des aînés dans la société québécoise.

À part les différences entre les générations, on peut comprendre le taux de suicide relativement faible des aînés du Québec à partir d'une analyse des facteurs de risque. Tout d'abord, si on conçoit le taux de suicide pour un sousgroupe d'une population comme étant fonction de la détresse psychologique, des problèmes de santé mentale et du bien-être général, nous possédons des données qui peuvent nous aider à comprendre la situation des aînés. Malgré certaines croyances populaires selon lesquelles les aînés constituent un groupe relativement défavorisé et malheureux au Québec, les résultats d'une enquête de Santé-Québec ${ }^{2}$ indiquent que, parmi tous les groupes d'âge, ce sont les personnes âgées qui vivent le moins de détresse psychologique, qui sont les plus satisfaites de leurs relations familiales, de leurs amis et de la qualité de leur vie. Leur niveau de satisfaction dans les rela- tions avec leur réseau social est le plus élevé parmi tous les groupes d'âge. Ceci indique qu'à l'encontre des croyances populaires, les aînés ne sont pas délaissés par leurs familles ni abandonnés par la société ou mécontents de leur vie en général. Il existe certainement des aînés qui sont très malheureux et qui souffrent de troubles de santé physique et mentale. Cependant, si on avait à dresser un portrait type du vieillissement au Québec, on constaterait que les personnes âgées sont généralement heureuses et qu'elles ont moins de problèmes d'isolement social que les jeunes.

\section{LES FACTEURS DE RISQUE}

La situation des aînés au Québec par rapport au suicide pose des défis pour les chercheurs qui s'intéressent à l'étiologie du suicide dans la société. Le bienêtre général des aînés et leur taux de suicide moins élevé semblent contredire le fait que ces personnes sont plus exposées à de nombreux facteurs de risque 
liés au suicide. En effet, en vieillissant on vit des pertes multiples ${ }^{3}$. Les aînés sont plus à risque de perdre un conjoint, de vivre des décès d'amis, des pertes d'emploi, des pertes de rôle parental, sans compter les pertes de capacité physique. Les recherches indiquent que toutes ces pertes sont liées à un risque élevé de suicide 4 . Les aînés ont plus de maladies chroniques et peuvent connaître plus de souffrance physique que d'autres groupes d'âge. Un autre facteur lié au risque suicidaire est la disponibilité des moyens pour se suicider. $\mathrm{Au}$ Québec, les aînés ont plus souvent en leur possession certains moyens de se suicider que les plus jeunes; la grande majorité des personnes âgées prennent des médicaments qu'elles pourraient utiliser pour s'enlever la vie (ou, dans certaines situations, elles peuvent arrêter de prendre les médicaments qui sont nécessaires pour leur survie).

Il faut aussi faire remarquer que la génération actuelle des aînés consulte moins souvent les professionnels de la santé mentale que les personnes plus jeunes. Les aînés d'aujourd'hui ont plus de préjugés qui font en sorte qu'ils évitent les professionnels de la santé mentale à cause de la stigmatisation attachée au fait d'être considéré comme «fou». De plus, ils téléphonent moins souvent aux centres de prévention du suicide parce qu'ils n'ont pas eu l'habitude de parler de leurs problèmes à des étrangers (nous parlons de personnes qui, dans certains cas, n'avaient même pas le téléphone chez elles lorsqu'elles étaient jeunes). Lorsque l'on considère la présence d'un si grand nombre de facteurs de risque $\mathrm{du}$ suicide, on peut se demander pourquoi les aînés du Québec ne se suicident pas plus que d'autres groupes d'âge.

La religion est souvent citée comme étant possiblement un facteur de protection du suicide chez les aînés. Il est certain que les aînés au Québec sont plus pratiquants que les générations plus récentes et que l'influence de l'Église dans la vie des Québécois a diminué énormément depuis la Révolution tranquille des années 1960. Cependant, les recherches réaliséesjusqu'à ce jour ne nous permettent pas de confirmer un lien entre le niveau de croyance ou de pratique religieuse et le suicide 5 .

\section{LE SUICIDE ET LA MALADIE PHYSIQUE}

Selon les croyances populaires, les personnes ayant atteint la phase terminale d'une maladie grave ou souffrant de troubles dégénératifs s'enlèvent souvent la vie pour abréger la souffrance physique ou psychologique associée à la maladie. En vieillissant, les risques d'être atteint d'une maladie chronique ou dégénérative augmentent et la majorité des êtres humains souffrent pendant la phase terminale de la maladie qui finalement les emporte. Cependant, malgré ces croyances populaires, le lien entre le suicide et les maladies chroniques, dégénératives ou en phase terminale n'est pas toujours présent: tout dépend de la nature de la maladie ainsi que des ressources $\mathrm{du}$ milieu. On trouvera dans Mishara ${ }^{6}$ une synthèse des recherches empiriques portant sur les facteurs qui influencent le désir des personnes atteintes d'une

\section{Mortalité par suicide au Québec,1997}

taux pour 100,000 par groupes d'âge

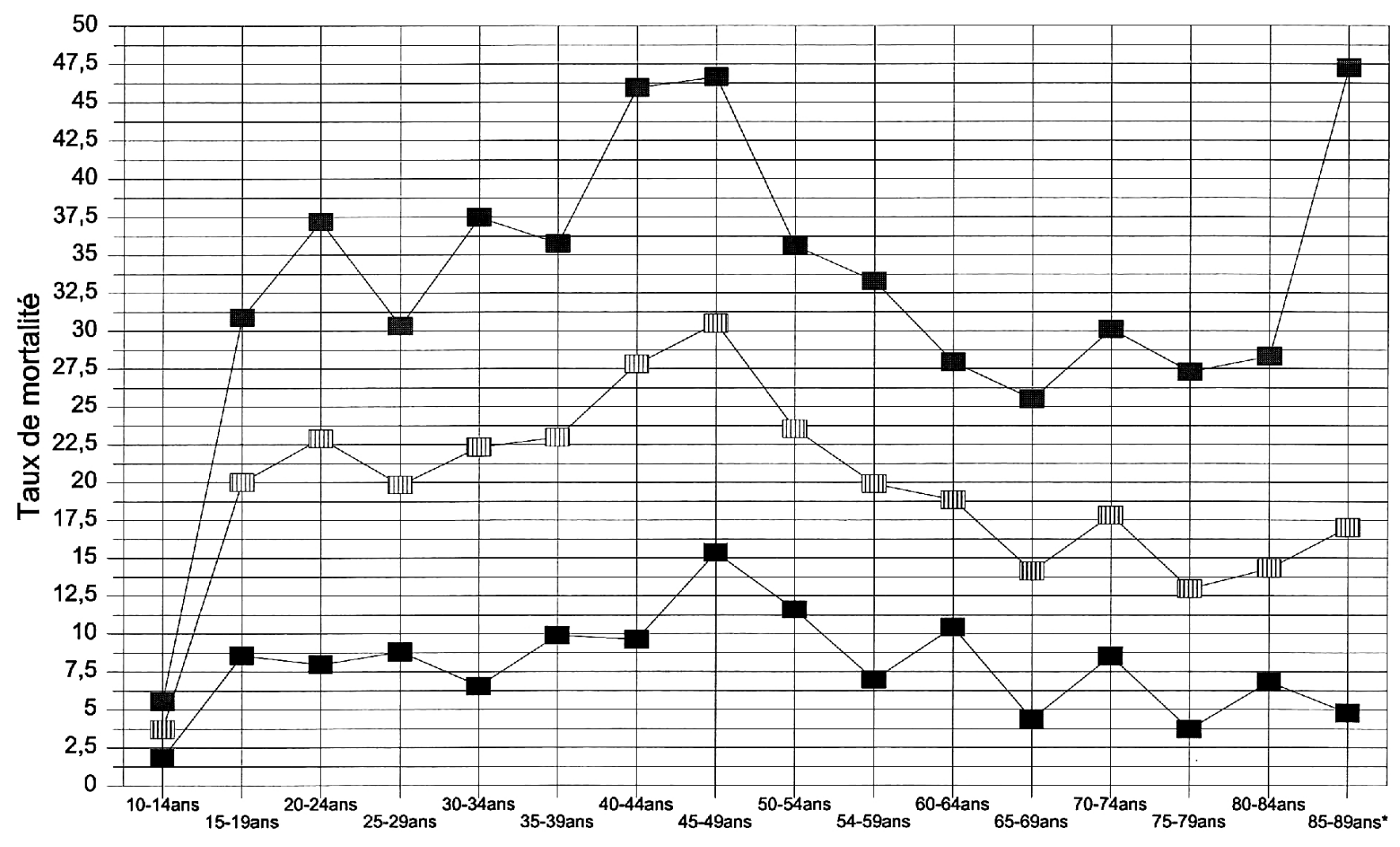

- Femme - Homme $-\mathbb{m}$ - Total 
maladie grave en phase terminale, ou souffrant d'une maladie chronique, de choisir une mort prématurée par suicide ou par euthanasie. On y conclut que le risque de suicide est plus élevé pour les individus atteints de certaines maladies, telles que l'épilepsie, les dommages cérébraux, la maladie de Parkinson, le sida et le cancer. Cependant, de nombreuses maladies chroniques ne sont pas associées à un risque plus élevé de suicide, par exemple la démence sénile, la sclérose en plaques et de nombreux autres handicaps physiques. Les facteurs de risque et les mécanismes qui expliquent les liens entre un risque élevé de suicide et des maladies physiques varient énormément. Dans certains cas (par exemple la chorée de Huntington), la dépression est une conséquence directe et physiologique de la maladie, et cette dépression augmente le risque de suicide. Dans d'autres cas, par exemple les maladies $\mathrm{du}$ foie et les dommages cérébraux, l'alcoolisme est un facteur important dans le développement de la maladie, et probablement l'alcoolisme augmente le risque de suicide. Dans d'autres situations, par exemple dans le traitement de l'hypertension et de certaines maladies cardio-vasculaires, la dépression peut être un effet secondaire des médicaments utilisés pour le traitement de ces maladies. Les recherches indiquent que la douleur physique est rarement liée à un risque élevé de suicide. L'exception importante est le cancer où la dépression et la douleur physique non contrôlée sont liées à un risque plus élevé de s'enlever la vie.

Les recherches consacrées aux facteurs qui augmentent ou diminuent le risque de suicide chez les personnes atteintes d'une maladie terminale ou chronique indiquent que, malgré un risque un peu plus élevé de suicide chez les personnes malades, les liens entre les maladies et le suicide sont complexes. Par exemple, les recherches suggèrent qu'un grand nombre de personnes atteintes de maladies terminales qui s'enlèvent la vie ont déjà fait des tentatives de suicide ou souffert d'une dépression clinique avant d'être malades. Nous pourrions conclure, à partir de la recension des écrits sur le suicide, la maladie grave en phase terminale et la maladie chronique, que les aînés devraient constituer une catégorie où le risque de suicide est un peu plus élevé à cause de la prévalence dans ce groupe de certaines maladies liées à un risque élevé de suicide. Cependant, le risque de suicide semble plus élevé chez les personnes plus jeunes atteintes des mêmes maladies et l'importance de la maladie physique

ON PEUT TOUT D'ABORD COMPRENDRE LE TAUX DE SUICIDE

RELATIVEMENT FAIBLE DES AîNÉS COMME ÉTANT LE RÉSULTAT

D'UNE CERTAINE SAGESSE ASSOCIÉE À L'ÂGE AVANCÉ.

comme facteur de risque de suicide semble être mineure en comparaison avec d'autres facteurs.

\section{COMPRENDRE LE NON-SUICIDE DES AÎNÉS: QU'EN EST-IL DE LA SAGESSE?}

Afin de mieux comprendre les taux de suicide moins élevés chez les aînés au Québec malgré les multiples facteurs de risque présents dans leur vie, il est opportun d'analyser notre compréhension du suicide en général. Une meilleure compréhension des raisons pour lesquelles il n'y a pas plus de suicides chez les aînés pourra peut-être nous amener à mieux comprendre comment prévenir le suicide auprès de la population en général, et nous aider aussi à trouver comment nous y prendre pour éviter les décès par suicide des personnes âgées au Québec. Dans cette section, nous allons discuter de certaines explications possibles du suicide et du non-suicide des aînés, et des implications pour la prévention.

\section{LA SAGESSE À L'ÂGE AVANCÉ}

On peut tout d'abord comprendre le taux de suicide relativement faible des aînés comme étant le résultat d'une certaine sagesse associée à l'âge avancé. Une peine d'amour à l'âge de 14 ans peut sembler être la fin du monde, alors que les pertes importantes plus tard dans la vie peuvent être mieux vécues du fait que l'aîné a appris à transiger avec les difficultés et les événements stressants. $\mathrm{Si}$ on accepte cette explication, quelles sont les implications pour notre compréhension de l'étiologie du suicide en général et du suicide des personnes âgées? Si les aînés qui ne suicident pas sont plus sages, est-ce que cela signifie que les personnes âgées qui se tuent sont moins sages ou ont moins d'habiletés à résoudre des problèmes? Si la sagesse protège les aînés québécois, est-ce que les personnes âgées ailleurs dans le monde sont pour autant moins sages? Il est possible que dans d'autres milieux d'autres facteurs de risque (par exemple, le nombre plus élevé d'aînés vivant sous le seuil de la pauvreté) diminuent les effets protecteurs de la sagesse.
Il est important ici de définir ce qu'est «la sagesse». Est-ce qu'il existe vraiment une sagesse qui augmente avec l'âge et quelles en sont les composantes? Dans leur résumé des recherches sur la sagesse et le vieillissement, B. Mishara et R. Riedel ${ }^{7}$ concluent que certains types de sagesse sont associés au vieillissement; il s'agit de la sagesse conçue comme une capacité de résoudre des problèmes de vie d'après la connaissance de différents contextes, valeurs et priorités ${ }^{8}$. C'est là un concept de la sagesse qui fait référence aux habiletés à résoudre les problèmes de la vie quotidienne et au concept de mécanisme d'adaptation (le coping), soit les différentes façons de réagir ou de maîtriser les problèmes stressants et les difficultés de la vie.

Si on accepte l'hypothèse que c'est la sagesse qui protège un grand nombre d'aînés du suicide, on peut conclure que le même genre de sagesse, c'est-à-dire la capacité de transiger avec des problèmes de la vie courante, pourrait protéger du suicide des personnes de n'importe quel groupe d'âge, et que les aînés qui se tuent ont peut-être moins d'habiletés leur permettant de s'adapter aux problèmes et au stress, comparativement à d'autres personnes âgées. Il existe un grand nombre d'études qui lient les mécanismes d'adaptation (coping) et les habiletés à résoudre des problèmes chez les jeunes qui ont des tendances suicidaires 9 . Cependant, il n'existe pas d'études comparables faisant état de liens entre ces habiletés et les tendances suicidaires chez les personnes âgées. Cette explication demeure cependant une hypothèse intéressante à vérifier.

Si la sagesse peut contribuer à prévenir le suicide, quelles sont les implications pour la prévention du suicide chez les aînés? Ces implications dépendent de la sagesse ou des mécanismes d'adaptation. Si la sagesse est simplement liée à l'accumulation d'expériences ou à des différences de personnalité, il ne s'agit pas d'une habileté qu'on peut facilement modifier ou apprendre. Mais si l'on croit que les êtres humains sont capables d'apprendre d'autres façons de résoudre des problèmes et de réagir à des situa- 
tions difficiles et stressantes, la prévention du suicide peut être liée aux programmes qui visent l'amélioration de ces habiletés. Chez les jeunes, il existe plusieurs projets-pilotes visant l'augmentation des habiletés mettant à contribution les membres de leur réseau personnel et les services d'aide pour régler les problèmes de la vie quotidienne. Un exemple d'un programme évalué en prévention primaire est le projet «Reaching Young Europe» (évalué récemment au Danemark) qui vise à augmenter les habiletés des jeunes au début de l'école primaire en vue de trouver des solutions pour régler leurs problèmes ${ }^{10}$. Si on est capable d'apprendre aux enfants comment mieux gérer leurs problèmes, on devrait être en mesure de créer des programmes pour augmenter la sagesse des aînés. Les recherches sur la capacité des aînés à apprendre de nouvelles habiletés basées sur leurs expériences indiquent clairement que les aînés peuvent apprendre aussi bien ou mieux que les personnes plus jeunes ${ }^{11}$.

\section{LES MALADIES MENTALES}

L'un des facteurs de risque le plus souvent associé au décès par suicide est l'état de la santé mentale. Par exemple, au Québec, A.D. Lesage et al. ${ }^{12}$ ont trouvé que $88 \%$ des personnes de 18 à 35 décédées par suicide ans ont pu être diagnostiquées comme étant atteintes d'un trouble mental. Dans cette même étude, le trouble mental identifié, dans $37 \%$ des cas, était une dépression majeure, alors que $23 \%$ des personnes avaient un problème de toxicomanie et $24 \%$, un problème d'alcoolisme. Les études consacrées au suicide chez les aînés indiquent qu'en grande majorité ces personnes souffraient d'un «désordre affectif», généralement une dépression clinique. Mais, en comparaison avec les jeunes, les désordres affectifs sont rarement associés à une personnalité antisociale, le diagnostic qui caractérise les délinquants, ou à l'abus de substances. En général, les recherches indiquent qu'il y a moins de symptômes de dépression chez les personnes âgées que chez les personnes plus jeunes ${ }^{13}$ et que le taux de dépression chez les aînés, ainsi que celui d'autres désordres, est moins élevé que chez les personnes plus jeunes ${ }^{14}$. Y. Conwell, M. Rotenberg et E.D. Caine15, dans une étude portant sur 246 décès par suicide de personnes ayant plus de 50 ans, ont trouvé moins souvent de désordres psychiatriques que dans les études d'échantillons de personnes plus jeunes. Ces faits peuvent expliquer le taux moins élevé de suicide chez les aînés du Québec. Cependant, cela n'explique pas le fait qu'il y ait plus de suicides chez les aînés ailleurs dans le monde.

Dans une recension récente de l'épidémiologie des désordres affectifs chez les aînés ${ }^{16}$, les auteurs examinent l'hypothèse selon laquelle les aînés ont un risque plus élevé de dépression à cause des changements biochimiques liés au vieillissement, ainsi qu'une augmentation des facteurs de risque tels que le deuil, les pertes psychologiques, les maladies somatiques et l'institutionnalisation. Les auteurs concluent que, malgré la présence de ces multiples facteurs de risque, les recherches indiquent que la dépression est plus élevée avant la prise de la retraite et relativement faible dans les 10 ou 15 ans qui suivent la retraite. Il y a une augmentation plus tard dans la vie, mais seulement après l'âge de 75 ans.

Jusqu'à maintenant, une petite proportion des aînés vivant dans la communauté est traitée avec des antidépresseurs ou avec des interventions psychothérapeutiques. Récemment, avec le développement de nouveaux antidépresseurs mieux tolérés par les personnes âgées, on constate une augmentation des prescriptions d'antidépresseurs auprès des aînés vivant dans la communauté. Cependant, on n'a pas encore d'indications permettant de savoir si cette augmentation de prescriptions d'antidépresseurs aux aînés est liée à une diminution de la dépression ou aux taux de suicide dans cette population.

Si on accepte l'idée selon laquelle les désordres affectifs, particulièrement la dépression clinique, sont souvent présents chez les aînés suicidaires, on a besoin d'effectuer des recherches afin de savoir si le traitement efficace de la dépression peut prévenir le suicide des personnes âgées. Jusqu'ici, les études auprès de personnes adultes indiquent que le traitement de la dépression par une combinaison de psychothérapie et de médicaments antidépresseurs est efficace pour prévenir des tentatives de suicide $^{17}$. Il importe maintenant d'effectuer des recherches semblables auprès des aînés.

\section{FACTEURS SOCIOÉCONOMIQUES}

Dans leur recension des recherches sur les facteurs de risque associés au suicide chez les personnes âgées, R.J. Dyck, B. Mishara et J. White ${ }^{18}$ constatent que le taux de suicide des aînés au Canada a diminué au cours des dix dernières années, ce qui coïncide avec une diminution du nombre de personnes âgées vivant sous le seuil de la pauvreté. Il appert donc que le maintien d'une bonne situation financière des aînés constitue un moyen de prévention possible du suicide. Dans la majorité des autres pays où les taux de suicide des aînés sont plus élevés, le pourcentage des aînés qui vivent sous le seuil de pauvreté est plus élevé.

Des chercheurs ont identifié des «facteurs de protection» liés à un risque moins élevé du suicide, ainsi que des facteurs de risque. R.J. Dyck, B. Mishara et J. White ${ }^{19}$ trouvent que le soutien social, en particulier le fait d'avoir un confident, contribue à réduire les comportements suicidaires chez les aînés. Cependant, la retraite, la perte ou le décès d'un conjoint ou d'un ami et le départ du foyer sont des facteurs de risque qu'on retrouve très souvent au sein de ce groupe d'âge. Un autre facteur en jeu dans le phénomène du suicide des aînés est l'attitude permissive de nombreux intervenants à l'égard du suicide chez les personnes âgées, qui fait en sorte que les aînés n'ont pas toujours les services d'intervention dont ils ont besoin.

Si un intervenant croit qu'il est «normal» ou «compréhensible» pour un aîné de s'enlever la vie, il aura tendance à ne pas vérifier si l'angoisse qui pousse la personne âgée à considérer le suicide peut être réduite par les interventions appropriées. B. Mishara et R. Riedel ${ }^{20}$, dans une recension des recherches sur les effets des interventions psychothérapeutiques auprès des personnes aînées, concluent que, malgré les croyances des intervenants selon lesquelles les personnes âgées sont difficiles à aider, celles-ci profitent autant ou plus des services offerts par les intervenants que les personnes plus jeunes.

\section{EN ATTENDANT LES RECHERCHES, QUOI FAIRE?}

Malgré le fait qu'il y ait moins de suicides chez les aînés que dans d'autres groupes d'âge, il y a quand même environ 200 Québécois âgés de 60 ans et plus qui s'enlèvent la vie chaque année. Qu'est-ce qu'on peut faire pour prévenir ces événements tragiques? Qu'est-ce qu'on peut faire pour prévenir une augmentation des suicides des aînés dans l'avenir, lorsque la génération actuelle des baby-boomers vieillira?

À un niveau sociétal, on peut continuer d'améliorer les conditions économiques et sociales des aînés dans notre société. La génération actuelle des aînés a eu relativement peu d'attentes à l'égard de la société lorsque ceux qui la 
constituent étaient jeunes. Depuis leur jeunesse, le Québec a développé un filet de sécurité dans les domaines de la santé et des services sociaux. Cependant, les personnes d'âge mûr aujourd'hui ont vécu la plus grande partie de leur vie avec une disponibilité de ces services. Leurs attentes sont donc plus élevées. Il est possible que les aînés auront dans l'avenir plus d'attentes quant aux rôles qu'ils veulent pouvoir jouer dans la société. Ils pourront être plus déçus de simplement se retrouver à la retraite et plus sujets à des angoisses et dépressions qui sont liées à un risque plus élevé du suicide.

Il est important que les sentinelles (médecins, prêtres, travailleurs sociaux, organisateurs des groupes d'âge d'or, etc.) reconnaissent le fait que les aînés puissent être suicidaires et qu'il est possible et souhaitable d'intervenir pour prévenir le suicide des aînés. La grande majorité de ceux-ci, même ceux souffrant de maladies physiques et de troubles de santé mentale, ne se tuent pas. Cependant, cela ne veut pas dire que certaines maladies physiques et les symptômes (par exemple, la douleur) ne créent pas des réactions d'angoisse et de dépression qui méritent l'attention des intervenants.

Le fait de vivre des pertes, d'avoir un désordre affectif ou d'autres facteurs de risque suicidaire ne sont pas des conditions suffisantes pour qu'une personne âgée s'enlève la vie et ne constituent pas des justifications pour un tel acte. Très souvent, les intervenants considèrent qu'il est moins important d'intervenir auprès des aînés parce que leur suicide est "plus justifié», ou parce que les aînés ne profiteront pas des interventions autant que les plus jeunes. Les recherches en gérontologie indiquent clairement que les aînés profitent autant des interventions psychothérapeutiques, voire mieux, que les autres groupes d'âge ${ }^{21}$. Les aînés suicidaires veulent rarement se tuer suite à un simple désir de mourir. Ils ressentent plutôt énormément de douleur psychologique et veulent se tuer pour mettre fin à leur souffrance. Il existe généralement d'autres façons de diminuer cette souffrance sans avoir recours à la mort. La dépression n'est pas liée au vieillissement normal, et le taux de succès des traitements de la dépression clinique chez les aînés est très élevé. Le défi pour les intervenants, ainsi que pour la société québécoise, est de trouver des moyens pour se défaire des préjugés qui font en sorte que les aînés n'obtiennent pas l'aide et l'appui dont ils ont besoin.

\section{Notes}

1 J.L. MCINTOSH, "Generational Analyses of Suicide: Baby-Boomers and 13ers», Suicide and Life-Threatening Behavior, vol. 24, no 4, 1994, p. 334-342.

2 C. BELLEROSE, C. LAVALLÉE, L. CHÉNARD et M. LEVASSEUR (dir. publ.), Et la santé, ça va en 1992-1993? Rapport de l'Enquête sociale et de santé 1992-1993, Montréal, Ministère de la Santé et des Services sociaux, Gouvernement du Québec, 1995.

3 B.L. MISHARA et R. RIEDEL, Le vieillissement (troisième édition révisée), Paris, Presses Universitaires de France, 1994.

4 SANTÉ CANADA, Le suicide au Canada: mise à jour du Rapport du Groupe d'étude sur le suicide au Canada, Ottawa, Santé Canada, 1994.

5 D. LESTER, Why People Kill Themselves: A 1990s Summary of Research Findings on Suicidal Behavior, 3e édition, Springfield, Ill., Charles Thomas Publisher, 1992.

6 B.L. MISHARA, «Synthesis of Research and Evidence on Factors Affecting the Desire of Terminally Ill or Seriously Chronically Ill Persons to Hasten Death", Omega, International Journal on Death and Dying, vol. 39, no 1, 1999, p. 1-70.

7 B.L. MISHARA et R. RIEDEL, Le vieillissement (troisième édition révisée), ibid.

8 P.B. BALTES et J. SMITH, «Toward a Psychology of Wisdom and its Ontogenesis» dans R.J. STERNBERG (dir. publ.), Wisdom. Its Nature, Origins and Development, Cambridge, University Press, 1990.

P.B. BALTES, U.M. STAUDINGER, A MAERCKER et J. SMITH, «People Nominated as Wise: A comparative Study of Wisdom-Related Knowledge», Psychology and Aging, vol. 10, no 2, 1995, p. 155-166.

9 A. SPIRITO, G. FRANCIS, J. OVERHOLSER et N. FRANK, "Coping, Depression and Adolescent Suicide Attempts», Journal of Clinical Child Psychology, vol. 25, no 2, 1996, p. 147155.

L. R. POLLOCK et J.M.G. WILLIAMS, Problem Solving and Suicidal Behavior, Suicide and Life-Threatening Behavior, vol. 28 , no 4, 1998, p. 375-387.

10 B.L. MISHARA et M. YSTGAARD, «Exploring the Potential of Primary Prevention: Evaluation of the Befrienders International Reaching Young Europe Pilot Pogramme in Denmark», (sous presse), Crisis.

11 B.L. MISHARA et R. RIEDEL, Le vieillissement (troisième édition révisée), ibid.

12 A.D. LESAGE et al., "Suicide and Mental Disorders: A Case-Control Study of Young Men», American Journal of Psychiatry, vol. 151, no 7, 1994, p. 10631068.

13 R.R. FRERICHS, C.A. ANESHENSEL et V.A. CLARK, Prevalence of Depression in Los Angeles County, Paper presented at the Society of Epidemiological Research, Minneapolis, Minnesota, 1980
14 R.C. BLAND, S.C. NEWMAN, S.C. et H. ORN, «Prevalence of Psychiatric Disorders in the Elderly in Edmonton, Acta Psychiatrica Scandinavica, no 77 (suppl. 338), 1988, p. 57-63.

15 Y. CONWELL, M. ROTENBERG et E.D. CAINE, "Completed Suicide at Age 50 and Over», Journal of the American Geriatric Society, no 38, 1990, p. 640644.

16 S. PALLSON et I SKOOG, " The Epidemiology of Affective Disorders in the Elderly: A Review», International Clinical Psychopharmacology, no 12 (suppl. 7), 1997, S3-S13.

17 K. HAWTON, "Controlled Studies of Psychosocial Interventions Following Attempted Suicide», dans N. KREITMAN et S.D. PLATT (dir. publ.), Current Research on Suicide and Parasuicide, Edinburgh, Edinburgh University Press, 1989.

18 R.J. DYCK, B.L. MISHARA et J. WHITE, Le suicide chez les enfants, les adolescents et les personnes âgées: constatations clées et mesures préconisées, dans Forum National sur la Santé: Les déterminants de la Santé, vol. 3: le cadre et les enjeux, Ottawa, Santé Canada, 1998, p. 323-390.

19 Ibid.

20 B.L. MISHARA et R. RIEDEL, Le vieillissement (troisième édition révisée), ibid.

21 Y. CONWELL, "Outcomes of Depression", The American Journal of Geriatric Psychiatry, no 4 (suppl. 1), 1996, S34S44. 